\title{
Imaging of 3D Cardiac Electrical Activity: A Model-Based Recovery Framework
}

\author{
Linwei Wang ${ }^{1}$, Heye Zhang ${ }^{1}$, Pengcheng Shi ${ }^{1,2}$, and Huafeng Liu $^{3}$ \\ ${ }^{1}$ Department of Electrical and Computer Engineering, \\ Hong Kong University of Science and Technology, Hong Kong \\ maomwlw, eeship@ust.hk \\ ${ }^{2}$ School of Biomedical Engineering, Southern Medical University, Guangzhou, China \\ ${ }^{3}$ State Key Laboratory of Modern Optical Instrumentation, Zhejiang University, \\ Hangzhou, China
}

\begin{abstract}
We present a model-based framework for imaging 3D cardiac transmembrane potential (TMP) distributions from body surface potential (BSP) measurements. Based on physiologically motivated modeling of the spatiotemporal evolution of TMPs and their projection to body surface, the cardiac electrophysiology is modeled as a stochastic system with TMPs as the latent dynamics and BSPs as external measurements. Given the patient-specific data from BSP measurements and tomographic medical images, the inverse problem of electrocardiography (IECG) is solved via state estimation of the underlying system, using the unscented Kalman filtering (UKF) for data assimilation. By incorporating comprehensive a priori physiological information, the framework enables direct recovery of intracardiac electrophysiological events free from commonly used physical equivalent cardiac sources, and delivers accurate, robust, and fast converging results under different noise levels and types. Experiments concerning individual variances and pathologies are also conducted to verify its feasibility in patient-specific applications.
\end{abstract}

\section{Introduction}

Noninvasive imaging or recovery of cardiac electrical activity from BSPs would be highly beneficial both as a clinical aid and a mass screening tool. Conventional strategies in IECG problem usually follow a typical pattern. First, a simplified physical equivalent source is used as implicit constraints on the problem, which is either surface projection or regional accumulation of the realistic electrical activity. Then as the central focus of most recent IECG investigations, mathematically motivated regularization is imposed as additional explicit constraints. In these approaches, difficulties in interpreting the relationship of the solution and underlying physiopathology are involved, and the lack of physiologically meaningful constraints remains the bottleneck that hinders the direct recovery scheme free from physical equivalent sources.

More fundamentally in terms of physiology, it is recognized that time-varying 3D TMP distributions not only preserves characteristic electrophysiological 
dynamics but also directly relates to alterations in BSPs. However, although remarkable achievements have been made in the forward simulation of BSPs from TMPs 12, little success has been achieved in the inverse direction since the problem is highly under-determined. We believe that adequate constraints are necessary and the incorporation of known electrophysiological information is a desired paradigm. Although an indirect recovery method based on optimization of heart excitation model parameters, the important work [3] shares with our intuition that rich a priori information from physiological models would ensure the meaningfulness of the constraints and reduce the difficulty in explicitly applying multiple constraints.

In this paper, we present a framework for directly recovering cardiac electrical activity in terms of 3D myocardial TMPs. In the framework, the system of electrophysiology is carefully modeled, and 3D cardiac electrical activity is recovered via state estimation of the underlying system. The dynamics of the system is described by the spatiotemporal evolution of TMPs and its relations to measurements by the TMP-BSP projection model. A stochastic state space interpretation of the system is then obtained. With the availability of patient-specific data from BSP measurements and heart-torso geometry from tomographic medical images, the recovery of TMPs is achieved using the sequential estimation technique, the unscented Kalman filtering (UKF), which is particularly suitable for our system with highly nonlinearity and complex system and data uncertainties beyond typical Gaussian assumption. Volumetric TMP map sequence is then generated to provide intuitive visualization of the patient-dependent electrophysiological dynamics throughout the entire cardiac cycle. Experiments concerning individual variances and pathologies are conducted to verify its accuracy, robustness, and feasibility in practical applications.

\section{Methodology}

\subsection{System Modeling of Cardiac electrophysiology}

Motivated by using the a priori physiological knowledge to constrain the IECG problem, we carefully model the nonlinear dynamic system of cardiac electrophysiology. The model-based system approach, on one hand, impose comprehensive physiological spatiotemporal constraints on the inverse problem simultaneously; on the other, BSP measurements and medical images (MRI and CT) from specific subjects are utilized complementarily to provide patientspecific information in the inverse process. The modeling work consists of 3 major components:

1. The patient-specific, image-derived heart-torso geometry model.

2. The nonlinear dynamic model describing spatiotemporal evolution of TMPs.

3. The projection model directly relating TMPs to BSPs.

It is worthy to point out that at current stage, we concentrante on illustrating the basic idea and rationales of the innovate recovery framework. Thus 
the modeling criteria emphasize adequate physiological constraints on the inverse recovery process, rather than realistic descriptions of electrophysiological phenomena. Without loss of generality, different models with varying complexity and differing specialization can be easily accommodated in the future work.

Image-derived heart-torso model. Patient-specific heart-torso model is constructed using geometry data extracted from tomographic medical images and proper numerical representations. Concerned with the intervention of myocardial anisotropy and inhomogeneity on intra-/extra-cardiac potential distribution [4, and its close relationship to pathology [2, the anisotropic and inhomogeneous heart model is used with mesh free representation, where the anisotropy is reflected via detailed fiber structure and the inhomogeneity via position-dependent electrical properties. In Contrast, since the inhomogeneity of the torso has little impact on BSP pattern [5], and experimental measurements [6] show that conductivity anisotropy is dominated by intracardiac tissue, the isotropic and homogeneous model based on surface (BEM) representation is a viable option.

Dynamic model for spatiotemporal evolution of 3D TMP. The dynamics of the electrophysiological system is described by the FithHugh-Nagumo-like action-diffusion equations since it maintains characteristic phenomenal properties of the dynamics of interests with computational feasibility.

With a Garlekin mesh free representation [7, the two-variable model can be written in compact form with matrices:

$$
\begin{gathered}
\frac{\partial U}{\partial t}=-M^{-1} K U+f(U, V) \\
\frac{\partial V}{\partial t}=b(U-d V) \\
f(U, V)=c_{1} U(1-U)(U-a)-c_{2} U V
\end{gathered}
$$

where $U$ is transmembrane potential field and $V$ for inhibition. The mass matrix $M$ is a function of material density and the stiff matrix $K$ is a function of the passive electrical conductivity. $f(U, V)$ describes the excitation where $a, b, c_{1}, c_{2}, d$ are parameters defining shapes of action potentials.

TMP-BSP projection model. The TMP-BSP projection model relates unobservable TMPs to noninvasive BSP measurements. In the quasi-static condition, the problem is viewed in a passive volume conductor with the source distributed only in the myocardium and the governing poisson equation with given boundary condition [1] is:

$$
\nabla \cdot(\sigma \nabla \phi)=-\nabla \cdot\left(\sigma_{h} \nabla u\right)
$$

where $\phi, u$ is for BSPs and TMPs, $\sigma_{h}, \sigma$ for intracardiac and extracardiac conductivity. Applying boundary integral method to equation(4) we get an equation with both surface and volume integral: 


$$
c(\xi) \phi(\xi)+\int_{\Gamma_{T}} \phi(x) q^{*}(\xi, x) \mathrm{d} \Gamma_{T}-\int_{\Omega_{H}}\left(\nabla \cdot\left(\sigma_{h} \nabla u\right)\right) \phi^{*}(\xi, x) \mathrm{d} \Omega_{H}=\int_{\Gamma_{T}} q(x) \phi^{*}(\xi, x) \mathrm{d} \Gamma_{T}
$$

where $\phi^{*}$ and $q^{*}$ are fundamental solutions with fixed forms. $\Gamma_{T}, \Gamma_{H}$ stands for the body and heart surface, and $\Omega_{H}$ for myocardium volume.

Commonly, the volume integral in equation(5) is approximated with simplified distributed dipoles [1. Alternatively we attempt an innovative mesh free approximation of the volume integral whereby 1), the use of simplified models is avoided; 2), through integral by part, the assumption which restricts TMPs within myocardium is automatically satisfied. To ensure unique solutions, constrains defining potential references are added [1] and the linear TMP-BSP relationship is established with Minimal Norm (MN) method:

$$
\Phi=\left(H_{a}^{T} H_{a}\right)^{-1} H_{a}^{T} B_{a} U=C U
$$

where $H_{a}, B_{a}$ are augmented forms of matrices $H, B$, where $H$ results from the boundary integral with the BEM and $B$ from volume integral with the mesh free method.

\subsection{D TMPs Recovery Through State Estimation}

Stochastic state space representation. By viewing TMPs as latent states and BSPs as external measurements, the electrophysiological system is represented in a state space representation. The dynamic model of 3D TMP evolution is transformed to the state equation and the TMP-BSP projection model to the measurement equation. Allowing for complex system and data uncertainties such as modeling errors, individual variances, system and measurement noises, which are too complicate to tackle with in the present study but too important to neglect, we rearrange equations $(1,2,6)$ into stochastic state space representation with state vector $X(t)=\left(U(t)^{T} V(t)^{T}\right)^{T}$, measurement $Y(t)=\Phi(t)$, noise terms $\omega(t), \nu(t)$ denoting uncertainties in dynamic and measurement models:

$$
\begin{gathered}
\frac{\partial X(t)}{\partial t}=\tilde{F}(X(t), \omega(t)) \\
Y(t)=\tilde{G}(X(t), \nu(t))
\end{gathered}
$$

where $\tilde{F}$ and $\tilde{G}$ represent functions from equations(1-3,6) after noises is incorporated 1 . Note that common additive Gaussian noise restrictions are not demanded, so complex uncertainties can be described in a more general way.

State estimation with the UKF. The system given by equations $(7,8)$ has continuous dynamics but discrete measurements, thus further temporal discretization is demanded. A fourth-order Runge-Kutta solver to equation(7) is embedded in the inverse process to fulfil the discretization implicitly for the sake of reasonable accuracy and numeric stability.

\footnotetext{
${ }^{1}$ Detailed derivation omitted due to space limitation.
} 


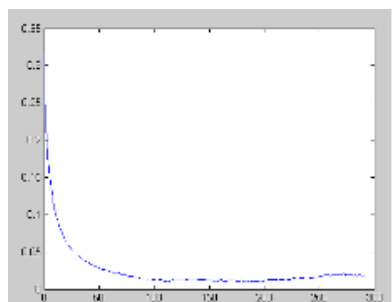

(a)

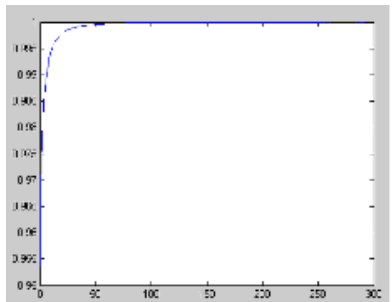

(b)

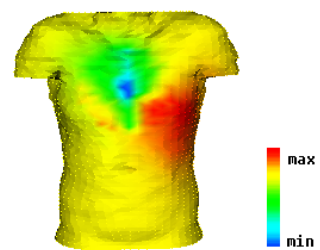

(c)

Fig. 1. Exemplary curves of RMSE (a) and CC (b) of recovery errors. (c), Exemplary BSP map at $140 \mathrm{~ms}$ used as input for recovery framework.

Concerned with the nature of the system under study, which comprises highly nonlinear models without explicit discretized form and complicate uncertainties beyond Gaussian assumptions, we use the UKF as the minimum mean least square (MMLS) estimator for the state estimation [8]. In brief, TMPs are estimated in a recursive manner with each new measurement $Y_{k}: 1$ ), deterministically generate a set of points (sigma points) $\left\{\mathcal{X}_{i}^{a}, W_{i}\right\}_{0}^{2 n}$ to approximate distribution of $X_{k-1}^{a}$ based on previous estimates; 2), sigma points are transformed individually through state equation (7) and the prior distribution of the state is obtained; 3 ), the transformed sigma points are further transformed through measurement equation (8) and the distribution of the new measurement and its cross-covariance with the state are computed; 4), with the real measurement, the posterior distribution of the state is obtained through the linear update in a KF context.

In practical implementation, to reduce the sampling non-local effects 9 caused by the large dimension and strong nonlinearity of our model, and to improve efficiency, enhance numerical stability and guarantee positive semi-definite covariance matrices 10, square-rooted, scaled UKF is utilized.

\section{3D Ventricular TMP Imaging: Results and Dicussion}

Experiments are conducted on a specific heart-torso model constructed from geometry data provided by [1112. In the present study, to assess and exemplify the capabilities of the recovery framework, all results are from experiments on the phantoms, where longs run of the perfect models $(7,8)$ are assumed to represent the ground truth. Observations are generated by adding noises of different types (Gaussian and Poisson) and different SNR levels (20dB and 30dB) to the true, downsampled BSPs. The generated BSP measurements are then feed back into the recovery framework, where the filtering technique is applied to estimate the latent dynamics of the electrophysiological system.

The resultant TMP map sequence represents complete cardiac electrical activity throughout the entire cardiac cycle. In early steps, individual variance or pathology is not considered. Later on, experiments concerned with patient-specific 
Table 1. RMSE, CC and maximum point-point errors of the recovery errors after convergence in terms of Mean \pm Deviance, with a initial value around 0.3 and 0.9 for RMSE and CC, and 0.5 for averaged scaled TMP value respectively

\begin{tabular}{|c|c|c|c|c|}
\hline \hline Error Metric & Gaussian(20dB) & Gaussian(30dB) & Poisson(20dB) & Poisson(30dB) \\
\hline RMSE & $0.0129 \pm 0.0022$ & $0.0114 \pm 0.0018$ & $0.0132 \pm 0.0033$ & $0.0123 \pm 0.0020$ \\
\hline CC & $0.9999 \pm \mathrm{e}-005$ & $0.9999 \pm \mathrm{e}-005$ & $0.9999 \pm \mathrm{e}-005$ & $0.9999 \pm \mathrm{e}-005$ \\
\hline Max.error & $0.0692 \pm 0.0050$ & $0.0522 \pm 0.0033$ & $0.0712 \pm 0.0058$ & $0.0664 \pm 0.0052$ \\
\hline \hline
\end{tabular}
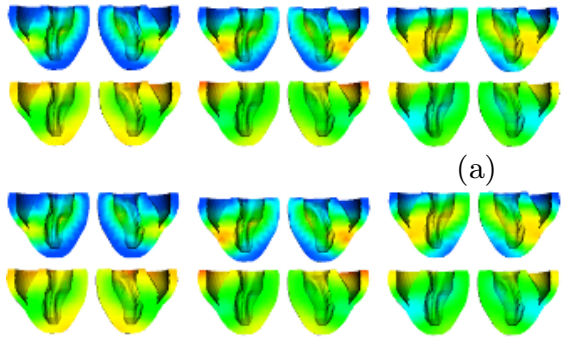

(b)

(a)
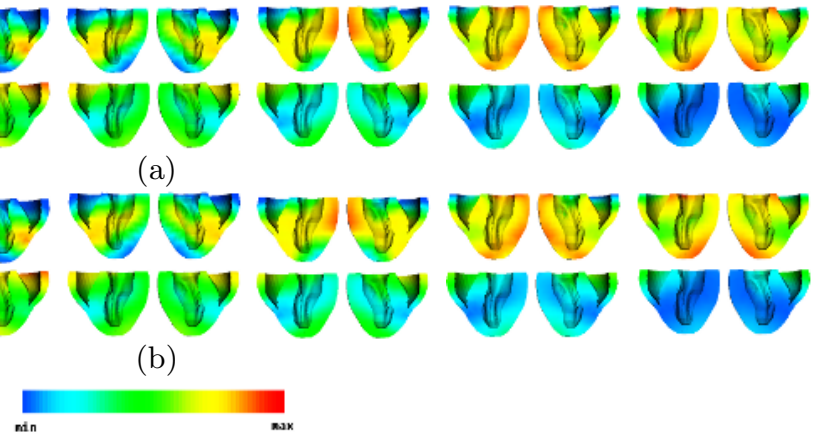
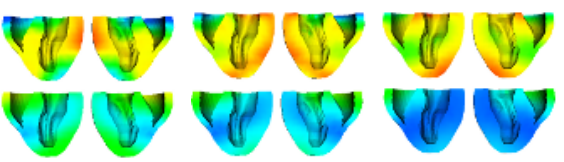

Fig. 2. Ventricular TMP map sequence of the ground truth (a) and the recovered results under 20dB Gaussian noise (b) at 12 time instances from $25 \mathrm{~ms}$ to $300 \mathrm{~ms}$ with $25 \mathrm{~ms}$ interval between. All TMP values are scaled and all color scales go from minimum(blue) to maximum (red). In both sequence, the spread of the green front in the first 4 maps corresponds to the fast depolarisation (activation propagation), the spread of the light blue front in the last 4 maps represents the slower repolarisation (recovery sequence), and those between correspond to the plateau of TMPs.

case are also implemented to test the applicability of our framework. Assess of results is presented both qualitatively through TMP imaging and quantitatively in terms of global RMSE (relative mean square errors), CC (correlation coefficients) and local point-point errors.

3D Ventricle TMP imaging of normal heart. In these initial experiments, both phantoms and the recovery employ models with standard parameters (set according to [13] in present study). Earliest activation sites are set according to experimental observation [14. An exemplary input BSP map is shown in Figure 1.c. Typical RMSE and CC curves are listed in Figure 1. $a$ and 1.b to give a rough example of the accuracy, fast convergence and consistent stability of results. The quantitative comparisons in Table. 1 illustrate the robustness of our framework to consistently provide accurate results under different disturbances. The recovered TMP map sequence against the true TMP map under 20dB Gaussian noises are presented in Figure 22. The recovered TMP map sequence is in good accordance

${ }^{2}$ The detailed interpretation is in the caption. 


\section{(1)}
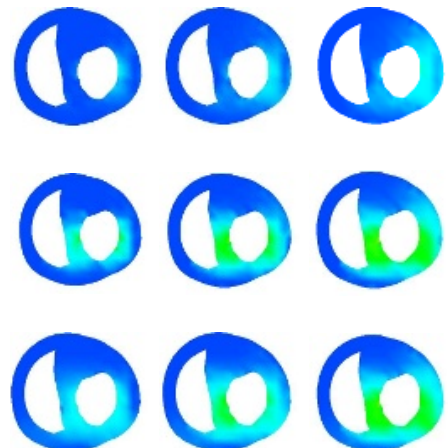
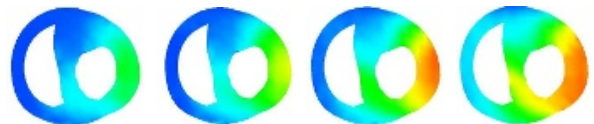

(a)
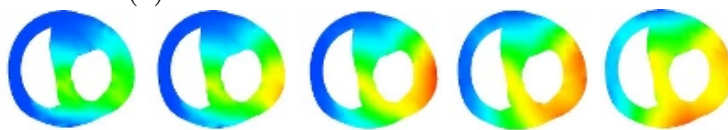

(b)
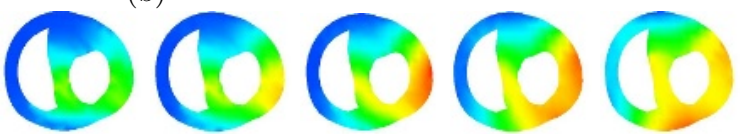

(c)

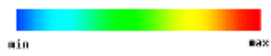

Fig. 3. TMP map sequence of cross section including the sites of ectopic focus at 8 time instances (Left to Right) from $15 \mathrm{~ms}$ to $120 \mathrm{~ms}$ with $15 \mathrm{~ms}$ interval between: (a) the normal heart, (b) the ground truth of pathology, (c) the recovered TMPs for pathology

with the true sequence, both in spatial distributions and temporal evolutions, thus the capability of the 3D TMP imaging to provide accurate, comprehensive and intuitive visualization of cardiac electrical activity is verified.

TMP imaging for patient-specific cases. One desirable performance of the framework is its feasibility in dealing with pathologies and individual variances in patient-specific applications. The pathology of ectopic focus, caused by additional impulse besides those from sinoatrial nodes, is tested. The forward simulation is initiated by introducing two additional excitation points while the inverse recovery make no use of such a prior knowledge. The resultant RMSE and CC between recovered TMPs and pathologic ground truth is 0.0227 and 0.9997 while with normal ground truth is 0.1126 and 0.8024 . Figure 3 lists the cross sectional map sequence of normal, pathologic and recovered TMP respectively. It verifies that though without a prior information, the recovery framework can not only timely and correctly reflect the cardiac abnormality, but also capture the site of ectopic focus. The future possibility of aiding clinical diagnosis can be expected.

Tests concerning individual variances are conducted with phantoms generated with slightly disturbed model parameters while the recovery implemented with standard ones. In an example considering conductivity variances, 3 , where longitudal component of $\sigma_{h}$ is deviated from standard values by $\pm 10 \%$ in phantom generation, the resultant RMSE and CC is $0.0122 \pm 0.0028$ and $0.9999 \pm \mathrm{e}-005$ respectively. These first results are encouraging in the feasibility of the framework in patient-specific applications.

${ }^{3}$ Multitude experiments have been implemented investigating the influences of individual variances, however due to space limitation, only exemplary results are given in this paper. 
Efforts focusing on improving the framework in patient-specific applications, primarily through automatically adjusting the generic model or its parameters to patient-specific data, and via data-fusion using electromechanical coupling, are under progress.

Acknowledgement. This work is supported in part by China National Basic Research Program (973-2003CB716100), Hong Kong Research Grants Council (CERG-HKUST6151/03E) and National Natural Science Foundation of China (60403040). For providing geometry data, we thank the Center for Integrated Biomedical Computing (CIBC) at the Univ. of Utah and the Bioengineering Institute of Auckland Univ. of Technology.

\section{References}

1. Aoki, M., Okamoto, Y., Musha, T., Harumi, K.: Three dimensional simulation of the ventricular depolarization and repolarization processes and body surface potentials: normal heart and bundle branch block. IEEE Trans.Biomed.Eng. 34 (1964) 454-461

2. Wei, D., Okazaki, O., Harumi, K., Harasawa, E., Hosaka, H.: Comparative simulation of excitation and body surface electrocardiogram with isotropic and anisotropic computer heart models. IEEE Trans.Biomed.Eng. 42 (1995) 343-357

3. He, B., Li, G., Zhang, X.: Noninvasive imaging of cardiac transmembrane potentials within three-dimdensional myocardium by means of a realistic geometry aniostropic heart model. IEEE Trans.Biomed.Eng. 50 (2003) 1190-1202

4. Roberts, D., Hersh, L., Scher, A.: Influence of cardiac fiber orientation on wavefront voltage, conduction velocity and tissue resistivity. Circ.Res. 44 (1979) 701-712

5. Rapport, B., Rudy, Y.: The inverse problem in electrocardiography: a model study of the effects of geometry and conductivity parameters on the reconstruction of epicardial pontetials. IEEE Trans.Biomed.Eng. 33 (1986) 667-675

6. Rush, S., Abildskov, J., R.McFee: Risistivity of body tissues at low frequencies. Circ.Res. 12 (1963) 40-50

7. Zhang, H., Shi, P.: A meshfree method for solving cardiac electrical propagation. In Ann.Int.Conf.IEEE EMBS (2005)

8. Julier, S., Uhlmann, J.: A new extension of the kalman filter to nonlinear systems. In Proc.of AeroSense:The 11th Int.Symp.on Aerospace/Defence Sensing,Simulation and Controls (1997)

9. Julier, S.: The scaled unscented transform. Int. J. Numer. Methods Eng. 47 (2000) $1445-1462$

10. Merwe, R., Wan, E.: The square-root unscented kalman filter for state and parameter-estimation. ICASSP 6 (2001) 3461-3464

11. Nash, M.: Mechanics and material properties of the heart using an anatomically accurate mathematical model. University of Auckland (1998)

12. MacLeod, R., Johnson, C., Ershler, P.: Construction of an inhomogeneous model of the human torso for use in computational electrocardiography. In 13th Ann.Int.Conf. IEEE EMBS. (1991) 688-689

13. Rogers, J., McCulloch, A.: A collocation-galerkin finite element model of cardiac action potential propagation. IEEE Trans.Biomed.Eng. 41 (1994) 743-757

14. Durrur, D., Dam, R., Freud, G., Janse, M., Meijler, F., Arzbaecher, R.: Total excitation of the isolated human heart. Comp. Methods Appl. Mech. Eng. 41 (1970) 899-912 